\title{
Efeito do tempo pós-prensagem da resina acrílica na alteração dimensional da base de prótese total
}

\section{Effect of acrylic resin post-pressing time on the dimensional alteration of complete denture base}

\author{
Rafael Leonardo Xediek CONSANI* \\ Saide Sarckis DOMITTI** \\ Lourenço CORRER SOBRINHO*** \\ Mário Alexandre Coelho SINHORETI***
}

\begin{abstract}
CONSANI, R. L. X.; DOMITTI, S. S.; CORRER SOBRINHO, L.; SINHORETI, M. A. C. Efeito do tempo pós-prensagem da resina acrílica na alteração dimensional da base de prótese total. Pesqui Odontol Bras, v. 15, n. 2, p. 112-118, abr./jun. 2001.
\end{abstract}

\begin{abstract}
A alteração dimensional da base de prótese total foi verificada em função do tempo pós-prensagem da resina acrílica. Foram confeccionados 20 corpos-de-prova constituídos por modelo de gesso/base de cera, os quais posteriormente foram incluídos pela técnica de rotina em muflas metálicas. A resina acrílica termopolimerizável Clássico foi proporcionada e manipulada de acordo com as instruções do fabricante. Após prensagem final sob carga de $1.250 \mathrm{kgf}$ em prensa de bancada, a resina acrílica foi submetida ao ciclo de polimerização em água aquecida a $74^{\circ} \mathrm{C}$ por 9 horas, segundo os tempos pós-prensagem imediato, 6, 12 e 24 horas. As bases de resina foram fixadas no modelo com adesivo instantâneo e o conjunto seccionado lateralmente nas regiões correspondentes à distal dos caninos (A), mesial dos primeiros molares (B) e palatina posterior (C). O desajuste entre base e modelo foi verificado com microscópio comparador em cinco posições para cada tipo de corte. Os resultados submetidos à análise estatística e ao teste de Tukey (5\%) mostraram que não houve diferença estatística significativa na comparação entre os tempos pós-prensagem imediato e de 6 horas, assim como entre os tempos de 12 e de 24 horas; entretanto, houve diferença significativa entre os grupos com prensagem imediata/após 6 horas e os grupos com prensagem após 12/24 horas.
\end{abstract}

UNITERMOS: Prótese total; Resinas acrílicas.

\section{INTRODUÇÃO}

A alteração dimensional da base da prótese resulta da contração do monômero durante a polimerização e das tensões liberadas no esfriamento da mufla ${ }^{4}$. Por outro lado, variações no processamento não podem alterar o padrão de comportamento dimensional da resina acrílica ${ }^{12}$, devido à diminuição do peso molecular da cadeia do polímero resultante ${ }^{25}$.

Entretanto, a magnitude da alteração dimensional da resina acrílica pode sofrer influência de alguns fatores, como o método de polimerização ${ }^{6}$, envolvendo tensões internas causadas pelos diferentes coeficientes de expansão térmica do gesso e resina ${ }^{29}$ e a espessura da base, com diferentes localizações dentro da mufla ${ }^{8,23,30}$, afetando a adaptação e estabilidade da prótese total ${ }^{3,14}$.

Nessas condições, apesar de a resina acrílica ser o material mais utilizado na confecção da prótese total, devido à inúmeras condições apreciáveis, apresenta as desvantagens da contração de polimerização e distorção. A contração devida à reação de polimerização não é uniforme, sendo mais pronunciada na região posterior do palato ${ }^{24} \mathrm{e}$ dificilmente poderá ser compensada após o processamento ${ }^{8}$. Por outro lado, a distorção é resultante do esfriamento e da remoção da base do modelo de gesso, ambos causando liberação de tensões induzidas durante o processamento ${ }^{7,17,21}$. Conseqüentemente, a combinação contração de polimerização e liberação de tensões diminui o nível de adaptação aos tecidos de suporte comprometendo a estabilidade da base 1,13,15,26,27 $^{\text {. }}$

Objetivando atenuar todas as variáveis que pudessem comprometer a estabilidade da base, diversas técnicas de processamento foram propostas

*Pós-graduando em Clínica Odontológica, Área de Prótese; **Professor de Prótese Total; ***Professores de Materiais Dentários Faculdade de Odontologia de Piracicaba da UNICAMP. 
CONSANI, R. L. X.; DOMITTI, S. S.; CORRER SOBRINHO, L.; SINHORETI, M. A. C. Efeito do tempo pós-prensagem da resina acrílica na alteração dimensional da base de prótese total. Pesqui Odontol Bras, v. 15, n. 2, p. 112-118, abr./jun. 2001.

em substituição ao tradicional banho de água aquecida $^{20}$, dentre elas, a energia por microondas $^{18}$, luz visivel ${ }^{21}$ e calor seco ${ }^{11}$.

Entretanto, pesquisas mais recentes continuam mostrando que a adaptação da base ao modelo permanece insatisfatória ${ }^{19}$, sofrendo influência da espessura da base ${ }^{23}$, da geometria do palato ${ }^{5}$ e dos diferentes ciclos de polimerização ${ }^{2}$.

Por outro lado, outro fato também interessante foi que a polimerização efetuada imediatamente ou 24 horas após a prensagem da resina acrílica não influenciava na magnitude da alteração dimensional da prótese total ${ }^{16}$.

Com base nessas considerações, seria conveniente estudar se o tempo decorrido da prensagem final até o início da polimerização permitiria que o acomodamento da resina na fase plástica liberasse as tensões induzidas pela prensagem da mufla e diminuísse a quantidade de monômero residual, ambos responsáveis, em parte, pela alteração dimensional das bases de prótese total.

\section{PROPOSIÇÃO}

O propósito deste trabalho foi verificar a alteração dimensional das bases de prótese total superior, polimerizadas pelo ciclo convencional em água aquecida a $74^{\circ} \mathrm{C}$ por 9 horas, sob influência da pós-prensagem nos tempos imediato, 6, 12 e 24 horas.

\section{MATERIAL E MÉTODO}

Para a confecção dos 20 corpos-de-prova foi utilizada a resina acrílica polimerizada termicamente Clássico (Artigos Odontológicos Clássico Ltda.).

A partir de um modelo padrão metálico, representativo de um maxilar com rebordos normais, sem irregularidades e retenções, foram obtidos moldes de silicona por adição (Elite Double). Os respectivos modelos foram confeccionados com gesso-pedra tipo III (Herodent), proporcionado e espatulado segundo recomendação do fabricante. Foram confeccionados vinte modelos, sobre os quais foram construídas bases em cera rosa (Wilson), com $2 \mathrm{~mm}$ de espessura.

Após plastificação e adaptação das lâminas sobre o modelo, o excesso de cera foi recortado e o limite periférico orientado até se obter a vedação das zonas correspondentes ao sulco vestibular.

Os corpos-de-prova (modelo de gesso/base de cera) foram incluídos em muflas metálicas (J. Safrany) pela técnica de rotina, depois de isoladas com vaselina sólida (Sidepal). Os gessos comum tipo II (Chaves Ltda.) e pedra tipo III (Herodent) foram proporcionados e espatulados de acordo com as recomendações dos fabricantes. Após 1 hora, as muflas foram colocadas em água em ebulição por 5 minutos, para plastificação da cera. Em seguida, foram abertas e as bases de cera removidas, sendo as superficies de gesso lavadas com solução de água aquecida e detergente líquido (ODD).

A resina acrílica foi preparada na proporção volumétrica de 3 partes de polímero/ 1 de monôme$\mathrm{ro}^{4}$. Ao atingir a fase plástica, foi retirada do pote de vidro, homogeneizada manualmente e inserida na mufla, interposta por uma folha de celofane umedecida com água. Após fechamento da mufla, a prensagem inicial foi efetuada sob carga lenta e gradual até atingir $800 \mathrm{kgf}$. Após abertura da mufla e eliminação da folha de celofane e dos excessos de resina, a prensagem final foi efetuada sob carga de $1.250 \mathrm{kgf}$, por 30 minutos.

Em seguida, as muflas foram transferidas para grampos de mola e a resina acrílica submetida ao ciclo de polimerização em água aquecida a $74^{\circ} \mathrm{C}$ durante 9 horas, segundo os grupos estudados, de acordo com o tempo decorrido da pós-prensagem da resina acrílica, ou seja, imediatamente, 6, 12 e 24 horas. Após esfriamento à temperatura ambiente, as muflas de cada grupo foram abertas e as bases retiradas dos modelos para remoção dos excessos e acabamento de rotina. Em seguida, as bases foram fixadas nos respectivos modelos com adesivo instantâneo (Super Bonder), com intenção de evitar o deslocamento durante o seccionamento $^{2,5,19}$.

O conjunto modelo-base foi cortado lateralmente (Figura 1) com serra manual, nas regiões correspondentes à distal dos caninos (A), mesial dos pri-

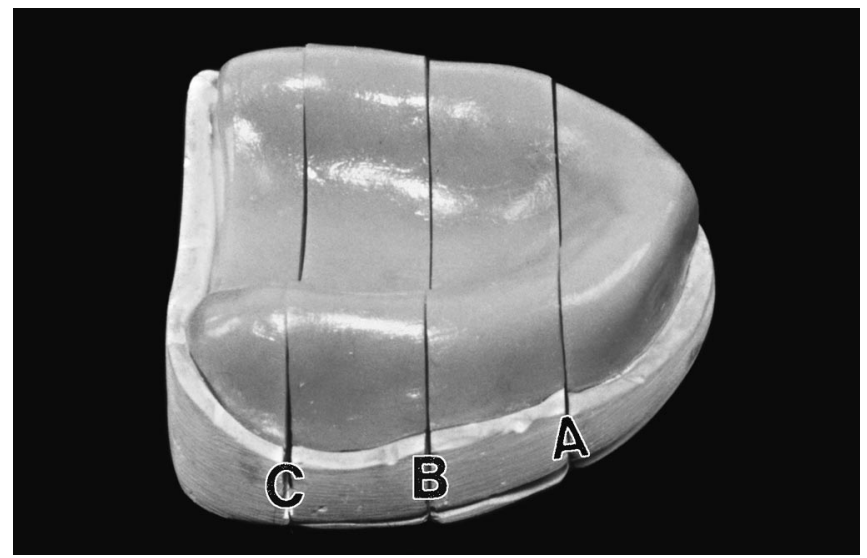

FIGURA 1 - Cortes laterais efetuados no conjunto modelo-base. 
CONSANI, R. L. X.; DOMITTI, S. S.; CORRER SOBRINHO, L.; SINHORETI, M. A. C. Efeito do tempo pós-prensagem da resina acrílica na alteração dimensional da base de prótese total. Pesqui Odontol Bras, v. 15, n. 2, p. 112-118, abr./jun. 2001.

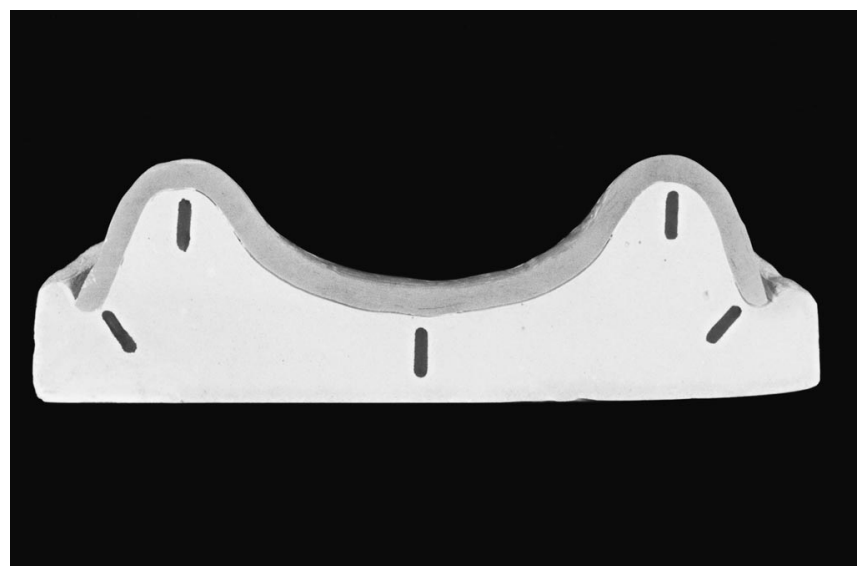

FIGURA 2 - Pontos onde foi avaliada a desadaptação da base de resina ao modelo de gesso $(8 \mathrm{X})$.

meiros molares (B) e zona palatina posterior (C), num dispositivo que posicionava e fixava o corpo-de-prova para a padronização dos cortes. A serra manual era movimentada lentamente, de maneira intermitente, com o propósito de não gerar calor durante o corte. Em cada seção, a adaptação base-modelo foi analisada nos pontos fundo do sulco vestibular direito, crista do rebordo direito, linha mediana, crista do rebordo esquerdo e fundo do sulco vestibular esquerdo (Figura 2), com microscópio comparador (Leitz), com leitura digital e precisão de 0,001 $\mathrm{mm}$.

\section{RESULTADOS}

Os dados obtidos nos grupos foram submetidos à análise estatística e ao teste de Tukey, em nivel de $5 \%$ de significância.

A Tabela 1 e o Gráfico 1 mostram as médias dos desajustes promovidos na base, constatanto-se a influência dos tempos pós-prensagem. Não houve diferença estatística significativa entre os valores dos grupos representados pelos tempos imediato e 6 horas, assim como entre os valores dos grupos com os tempos de 12 horas e 24 horas; entretanto, os grupos imediato/ 6 horas e 12/24 horas mostraram diferença significativa entre si. Os menores valores foram apresentados pelos tempos pósprensagem 12 horas e 24 horas.

Quando o fator corte foi analisado (Tabela 2 e Gráfico 2), verificou-se o mesmo comportamento de desajuste nos três cortes, isto é, não houve diferença estatística significativa entre os grupos pósprensagem imediato/ 6 horas e entre os grupos 12 horas/24 horas, com exceção do corte A no tempo 6 horas, que mostrou semelhança estatística com os demais períodos pós-prensagem.
Por outro lado, a Tabela 3 e o Gráfico 3 mostram que todos os tempos pós-prensagem promoveram desajustes entre os cortes com diferença estatística significativa, sendo a menor desadaptação apresentada no corte A e a maior no $\mathrm{C}$, ficando o corte B com valores intermediários.

\section{DISCUSSÃO}

A Tabela 1 e o Gráfico 1 mostram que os niveis de adaptação das bases de prótese total foram influenciados pelos diferentes tempos pós-prensagem da resina acrílica. Apesar de não haver diferença estatística significativa entre os valores obtidos com os tempos imediato e de 6 horas e entre os valores obtidos com os tempos de 12 horas e 24 horas, a comparação entre os valores obtidos com os tempos imediato/ 6 horas e os obtidos com os tempos 12/24 horas mostra que esses são estatisticamente diferentes entre si. Os menores desajustes foram obtidos nos tempos pós-prensagem de 12 horas e 24 horas.

Mesmo considerando-se que não tenha havido diferença estatística significativa entre os valores

TABELA 1 - Médias dos desajustes (mm) da base sob influência do tempo pós-prensagem.

\begin{tabular}{c|c|c}
\hline \hline Tempo pós-prensagem & Média do desajuste & Tukey $(5 \%)$ \\
\hline Imediato & $0,210(0,054)$ & $\mathrm{a}$ \\
\hline 6 horas & $0,200(0,069)$ & $\mathrm{a}$ \\
\hline 12 horas & $0,142(0,021)$ & $\mathrm{b}$ \\
\hline 24 horas & $0,141(0,020)$ & $\mathrm{b}$ \\
\hline \hline
\end{tabular}

Médias seguidas por letras distintas diferem entre si. Diferença média significativa $=0,023$. Desvio-padrão entre parênteses.

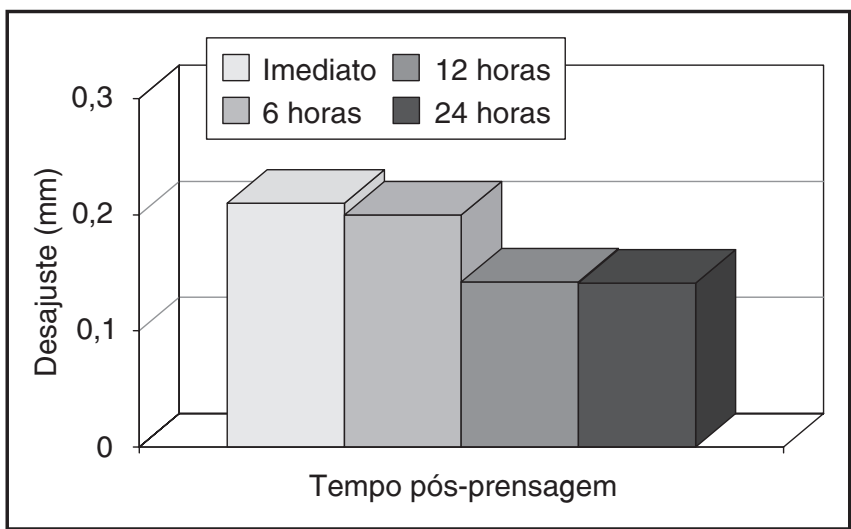

GRÁFICO 1 - Representação gráfica das médias dos desajustes $(\mathrm{mm})$ da base sob influência do tempo pósprensagem. 
CONSANI, R. L. X.; DOMITTI, S. S.; CORRER SOBRINHO, L.; SINHORETI, M. A. C. Efeito do tempo pós-prensagem da resina acrílica na alteração dimensional da base de prótese total. Pesqui Odontol Bras, v. 15, n. 2, p. 112-118, abr./jun. 2001.

de desajustes produzidos com os tempos pósprensagem imediato e de 6 horas, assim como entre os valores obtidos com os tempos de 12 horas e 24 horas, existe forte indício que a demora para o início da polimerização exerce influência benéfica na estabilidade dimensional das bases de resina acrílica.

Mesmo que KIMPARA; MUENCH ${ }^{16}$ (1996) não tenham observado influência do tempo de espera para polimerização na magnitude da alteração dimensional da prótese total, os nossos resultados mostram que o efeito do tempo pós-prensagem fica mais evidente quando se verifica que os tempos mais próximos da prensagem da resina acrílica (imediato e 6 horas) produziram maiores índices de desajuste quando comparados aos obtidos pelos tempos mais distantes (12 e 24 horas). A influência da pós-prensagem sobre os valores de desajuste se estabelece por meio do período de tempo que permite melhor acomodamento da resina no interior da mufla, possibilitando a diminuição dos niveis de monômero residual e o relaxamento das tensões impostas à massa, durante a prensagem do material resinoso.

A liberação do resíduo de monômero e o relaxamento das tensões impostas à resina podem ser considerados processos morosos, tendo em vista que não houve diferença estatística significativa entre os valores dos tempos pós-prensagem imediato e 6 horas.

Essa hipótese pode ser reforçada pelo fato de os valores obtidos nos tempos de 12 e 24 horas também não apresentarem diferença estatística significativa, talvez pelo esgotamento da liberação do monômero residual e do relaxamento das tensões, cuja identificação pontual não foi possivel devido às condições da metodologia, que estabeleceram

TABELA 2 - Médias dos desajustes (mm) da base para cada tempo pós-prensagem, dentro do fator corte.

\begin{tabular}{c|c|c|c}
\hline \hline \multicolumn{4}{c}{ Média do desajuste } \\
\hline \multirow{2}{*}{ Tempo } & $\mathrm{3}$ & $\mathrm{3}$ & Corte \\
\cline { 2 - 4 } & $\mathrm{A}$ & $\mathrm{B}$ \\
\hline Imediato & $0,164(0,005) \mathrm{a}$ & $0,185(0,018) \mathrm{a}$ & $0,281(0,007) \mathrm{a}$ \\
\hline 6 horas & $0,135(0,018) \mathrm{ab}$ & $0,197(0,042) \mathrm{a}$ & $0,268(0,061) \mathrm{a}$ \\
\hline 12 horas & $0,130(0,004) \mathrm{b}$ & $0,126(0,004) \mathrm{b}$ & $0,170(0,005) \mathrm{b}$ \\
\hline 24 horas & $0,130(0,004) \mathrm{b}$ & $0,124(0,004) \mathrm{b}$ & $0,170(0,005) \mathrm{b}$ \\
\hline \hline
\end{tabular}

Médias seguidas por letras distintas na coluna diferem em nivel de 5\%. Diferença média significativa $=0,041$. Desvio-padrão entre parênteses. um intervalo de 6 horas de demora entre os tempos dos grupos pós-prensagem. Essa situação mostra que o tempo ideal de espera pós-prensagem deverá ficar entre 12 e 24 horas, intervalos que apresentaram menores valores de desajuste, quando comparados aos obtidos nos períodos imediato e 6 horas.

Os resultados deste trabalho confirmam as recomendações de que a resina acrílica deve ficar descansando por 15 horas $^{9}$ ou por um tempo não inferior a 12 horas para não apresentar alterações dimensionais indesejáveis ${ }^{10}$.

Portanto, mesmo que muitas outras variáveis possam influenciar a magnitude das alterações dimensionais ocorridas na prótese total ${ }^{8}$, dentre elas a localização da espessura da base (zona central ou periférica) e o tempo de polimerização ${ }^{30}$, com certeza, a combinação de todos esses fatores indesejáveis pode diminuir o nivel de adaptação da base da prótese ao rebordo de suporte ${ }^{27}$. Por outro lado, o tecido mucoso bucal, mesmo mostrando razoáveis propriedades de adaptação, não compensaria desajustes além de $1 \mathrm{~mm}$, principalmente quando ocorrem na porção central da borda posterior palatina ${ }^{22}$.

Quando o fator tipo de corte foi analisado (Tabela 2 e Gráfico 2), verificou-se o mesmo padrão de desajuste nos cortes A, B e C, sem diferença estatística significativa entre os valores obtidos com os tempos pós-prensagem imediato e de 6 horas, ou entre os valores obtidos com os tempos de 12 horas e 24 horas, com exceção do corte A no tempo 6 horas, que mostrou semelhança estatística com os demais tempos. Os menores desajustes para os demais cortes foram mostrados nos tempos de 12 horas e 24 horas.

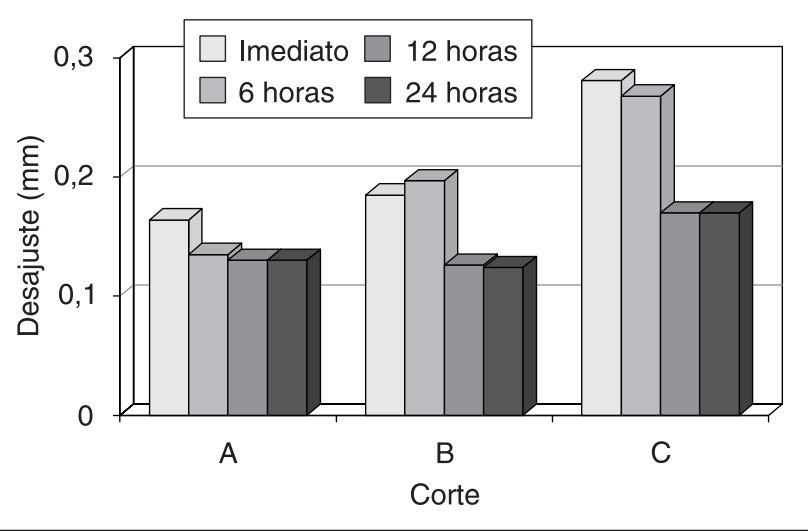

GRÁFICO 2 - Representação gráfica das médias dos desajustes $(\mathrm{mm})$ da base para cada tempo pós-prensagem, dentro do fator corte. 
CONSANI, R. L. X.; DOMITTI, S. S.; CORRER SOBRINHO, L.; SINHORETI, M. A. C. Efeito do tempo pós-prensagem da resina acrílica na alteração dimensional da base de prótese total. Pesqui Odontol Bras, v. 15, n. 2, p. 112-118, abr./jun. 2001.

TABELA 3 - Médias dos desajustes ( $\mathrm{mm}$ ) da base para cada tipo de corte, independentemente do fator tempo pós-prensagem.

\begin{tabular}{c|c|c}
\hline \hline Corte & Média do desajuste & Tukey $(5 \%)$ \\
\hline A & $0,140(0,017)$ & $\mathrm{a}$ \\
\hline B & $0,158(0,040)$ & $\mathrm{b}$ \\
\hline $\mathrm{C}$ & $0,222(0,061)$ & $\mathrm{c}$ \\
\hline \hline
\end{tabular}

Médias seguidas por letras distintas diferem entre si. Diferença média significativa $=0,030$. Desvio-padrão entre parênteses.

Neste caso, os cortes identificam padrões semelhantes de desajuste da base, mantendo a mesma relação estatística mostrada na Tabela 1 e no Gráfico 1. Esta situação parece confirmar as influências da espessura ${ }^{8,28}$ e da forma geométrica da base ${ }^{5}$ na magnitude e localização das distorções, fazendo acreditar que as demais variáveis do processamento não exerceram efeito crítico que pudessem modificar ou anular as possiveis alterações decorrentes dos tempos pós-prensagem.

Neste caso existe uma exceção, mostrada no corte A no tempo pós-prensagem de 6 horas, cujos resultados apresentam semelhança estatística com os obtidos pelos demais tempos. Este fato significa um resultado isolado, que mostra tendência do tempo 6 horas em apresentar valores sem diferença estatística quando comparado aos mostrados nos tempos imediato e 12 horas. Essa tendência talvez se justifique, quando se considera que a região abrangida pelo corte $\mathrm{A}$ apresenta condições topográficas restritivas, que dificultam a liberação de tensões após a remoção da base do modelo de gesso. Valores semelhantes de desadaptação obtidos nessa região por outros autores, quando estudaram a influência dos ciclos de polimerização ${ }^{2}$, da forma do palato ${ }^{5}$ e da resina acrílica $\mathrm{QC}-20^{19}$, parecem condições que também podem reforçar a influência restritiva da região anterior da base.

O fator corte também destacou outro dado importante, mostrado na Tabela 3 e no Gráfico 3. Independentemente dos demais fatores, os desajustes evidenciados pelos cortes A, B e C mostram alterações dimensionais similares às encontradas na literatura ${ }^{2,5,19}$. Isto significa que apesar da influência marcante dos tempos pós-prensagem, principalmente os de 12 e 24 horas, as condições impostas pela liberação do monômero residual e do relaxamento da resina antes da polimerização

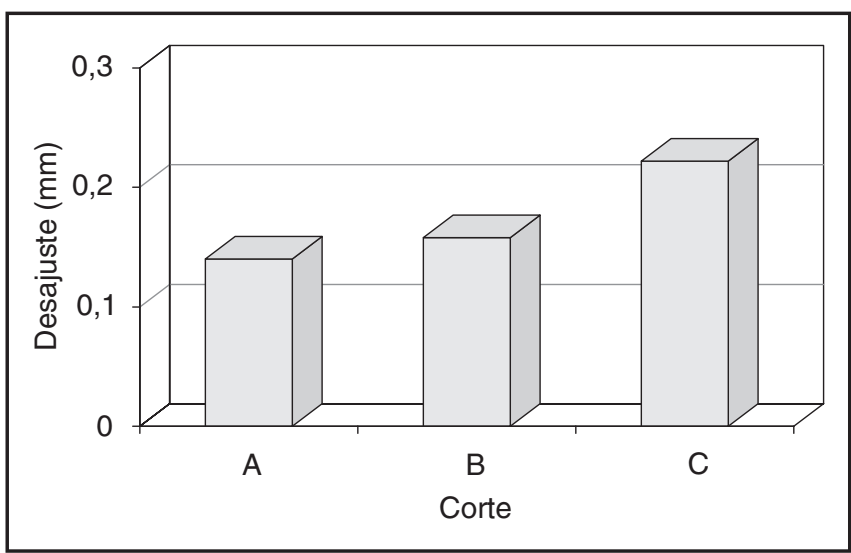

GRÁFICO 3 - Representação gráfica dos desajustes $(\mathrm{mm})$ da base para cada tipo de corte, independentemente do fator tempo pós-prensagem.

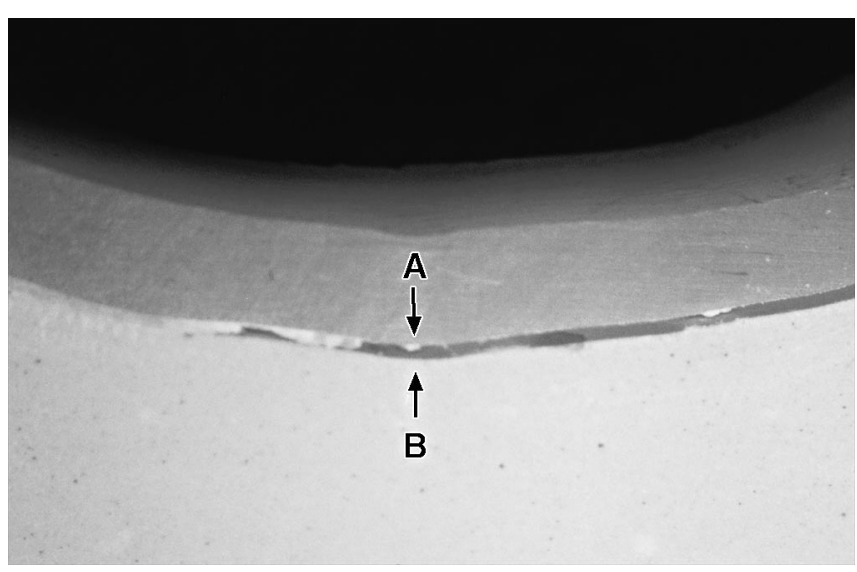

FIGURA 3 - Desajuste da base (A) ao molde de gesso (B) na região palatina mediana posterior $(8 \mathrm{X})$.

não foram suficientes para eliminar ou modificar o padrão de distorção da base.

O efeito do tempo pós-prensagem exerceu influência na magnitude dos valores da alteração dimensional, mostrada em todos os cortes. Entretanto, o padrão de distorção, evidenciando menor desajuste no corte A e maior no C, ficando o B com valores intermediários, todos com diferença estatística significativa, ocorre também devido às restrições impostas pela anatomia do $\operatorname{modelo}^{25} \mathrm{e}$ pela forma topográfica da região anterior do arco, que dificultam a liberação das tensões; enquanto a região palatina posterior, considerada zona mais plana e menos restritiva, favorece a liberação total das tensões causando distorção da base ${ }^{29}$.

Portanto, o padrão de distorção da base encontrado neste trabalho, onde o desajuste da linha média da região posterior palatina (Figura 3) foi maior que em outras áreas, com certeza comprova 
CONSANI, R. L. X.; DOMITTI, S. S.; CORRER SOBRINHO, L.; SINHORETI, M. A. C. Efeito do tempo pós-prensagem da resina acrílica na alteração dimensional da base de prótese total. Pesqui Odontol Bras, v. 15, n. 2, p. 112-118, abr./jun. 2001.

investigações anteriores ${ }^{3,21,22,30}$ e não foi modificada pelo tempo pós-prensagem da resina acrílica.

\section{CONCLUSÃO}

O nível de adaptação da base foi influenciado pelos tempos pós-prensagem, onde as menores médias de desajustes foram mostradas nos tempos 12 horas e 24 horas. Independentemente da magnitude da alteração dimensional, o padrão de distorção verificado na região palatina posterior da base foi confirmado no corte $\mathrm{C}$, por todos os tempos pós-prensagem. CONSANI, R. L. X.; DOMITTI, S. S.; CORRER SOBRINHO, L.; SINHORETI, M. A. C. Effect of acrylic resin post-pressing
time on the dimensional alteration of complete denture base. Pesqui Odontol Bras, v. 15, n. 2, p. 112-118,
abr./jun. 2001 .

The dimensional alterations of denture bases were verified in function of the acrylic resin post-pressing time. Twenty stone cast/wax base sets were confected for routine flasking procedure. Thermosetting acrylic resin (Clássico) was prepared according to the instructions of the manufacturer. After final pressing, the acrylic resin was submitted to polymerization in water at $74^{\circ} \mathrm{C}$ during 9 hours, following the immediate, 6-, 12-, and 24-hour post-pressing times. The resin bases were fixed on the casts with instantaneous adhesive and the sets were laterally sectioned in the regions corresponding to the distal aspect of canines (A), mesial aspect of first molars (B), and posterior palatal zone (C). The gap between the stone cast and the resin base was measured with a comparative microscope at five referential positions for each kind of sectioning. Data submitted to ANOVA and Tukey's test showed that there was no statistically significant difference between the immediate and the 6-hour post-pressing times as well as between the 12- and the 24-hour post-pressing times. However, there was statistically significant difference between the immediate/6-hour groups and the 12-/24-hour groups.

UNITERMS: Denture, complete; Acrylic resins.

\section{REFERÊNCIAS BIBLIOGRÁFICAS}

1. Al-HANBAli, E.; Kelleway, J. P.; HOWLETT, J. A. Acrylic denture distortion following double processing with microwave or heat. J Dent, v. 19, n. 3, p. 176-180, Mar. 1991

2. ALMEIDA, M. H. W.; DOMITTI, S. S.; CONSANI, S. et al. Influência de técnicas de polimerização sobre a adaptação das bases de prótese total. Rev Fac Odontol Passo Fundo, v. 4, n. 1, p. 49-56, jan./jun. 1999.

3. ANTHONY, D. H.; PEYTON, F. A. Dimensional accuracy of various denture base materials. J Prosthet Dent, v. 12, n. 1, p. 67-81, Jan./Feb. 1962.

4. ANUSAVICE, K. J. Phillips Materiais Dentários. 10. ed. Rio de Janeiro : Guanabara Koogan, 1998. 412 p.

5. ARIOLI FILHO, J. N.; DOMITTI, S. S.; CONSANI, S.; MOLLO JUNIOR, F. A. Influência da geometria do palato na adaptação de próteses totais superiores. Rev Fac Odontol Passo Fundo, v. 4, n. 1, p. 45-48, jan./jun. 1999.

6. BECKER, C. M.; SMITH, D. E.; NICHOLLS, J. I. The comparison of denture base processing techniques. Part II - dimensional changes due to processing. J Prosthet Dent, v. 37, n. 4, p. 450-459, Apr. 1977.

7. CARVALHO, J. C. M. Alterações dimensionais sofridas pela resina acrilica da base de aparelhos protéticos totais. Rev Fac Odontol São Paulo, v. 10, n. 1, p. 127-132, jan./jun. 1972 .

8. CHEN, J. C.; LACEFIELD, W. R.; CASTLEBERRY, D. J. Effect of denture thickness and curing cycle on the dimensional stability of acrylic resin denture bases. Dent Mater, v. 4, n. 1, p. 20-24, Apr. 1988.
9. CORREAA, G. A. Prótese total híbrida. São Paulo : Santos, 1996. $133 \mathrm{p}$.

10. GOMES, T.; MORI, M.; CORREAA, G. A. Atlas de caracterização em prótese total e prótese parcial removivel. São Paulo : Santos, 1998. 65 p.

11. GAY, W. D.; KING, G. E. An evaluation of the cure of acrylic resin by three methods. J Prosthet Dent, v. 42, n. 4, p. 437-440, Oct. 1979 .

12. HARMAN, I. M. Effects of time and temperature on polymerization of a methacrylate resin denture base. J Am Dent Assoc, v. 38, n. 2, p. 188-203, Feb. 1949.

13. HAYDEN, W. J. Flexural strength of microwave-cured denture baseplate. Gen Dent, v. 34, n. 5, p. 367-371, Sept./Oct. 1986.

14. JACKSON, A. D.; GRISIUS, R. J.; FENSTER, R. K.; LANG, B. R. The dimensional accuracy of two denture base processing methods. Int $\mathbf{J}$ Prosthod, v. 2, n. 5, p. 421-428, Sept./Oct. 1989.

15. KERN, W. R. Possible dimensional changes in denture base materials. J Am Dent Assoc, v. 28, n. 6, p. 1952-1958, Dec. 1941.

16. KIMPARA, E. T.; MUENCH, A. Influência de variáveis de processamento na alteração de dentaduras de resina acrílica. Rev Pos-Grad, v. 3, n. 2, p. 110-114, abr./jun. 1996.

17. MATHEWS, E. Residual problems in full denture prosthesis. Br Dent J, v. 97, n. 7, p. 167-177, Oct. 1954.

18. NISHII, M. Studies on the curing of denture base resins with microwave irradiation: with particular reference to heat-curing resins. J Osaka Dent Univ, v. 2, n. 1, p. 23-40, Feb. 1968.

19. PADOVAN, S. H. M.; DOMITTI, S. S.; CONSANI, S. Influên- 
CONSANI, R. L. X.; DOMITTI, S. S.; CORRER SOBRINHO, L.; SINHORETI, M. A. C. Efeito do tempo pós-prensagem da resina acrílica na alteração dimensional da base de prótese total. Pesqui Odontol Bras, v. 15, n. 2, p. 112-118, abr./jun. 2001.

cia dos ciclos de polimerização sobre a adaptação das bases de prótese total confeccionadas com resina acrílica QC-20. Salusvita, v. 18, n. 2, p. 73-88, mar. 1999.

20. PEYTON, F. A. Packing and processing dentures base resins. J Am Dent Assoc, v. 40, n. 5, p. 520-528, May 1950.

21. PHILLIPS, R. W. Skinner Materiais Dentários. 9. ed. Rio de Janeiro : Guanabara Koogan, 1993. 334 p.

22. POLYZOIS, G. L.; KARKAZIS, H. C.; ZISSIS, A. J. et al. Improving the adaptation of denture base by ancorage to the cuts: a comparative study. Quintessence Int, v. 21 , n. 3, p. 185-190, May 1990

23. SADAMORI, S.; GANEFIYANTI, T. D. G. R.; HAMADA, T. et al. Influence of thickness and location on the residual monomer content of denture base cured by three processing methods. J Prosthet Dent, v. 72, n. 1, p. 19-22, July 1994.

24. SKINNER, E. W. Acrylic resins: an appraisal of their use in dentistry. J Am Dent Assoc, v. 39, n. 3, p. 261-268, Dec. 1949.

25. SWEEney, W. T.; PAFFEnBARGER, G. C.; BEALL, J. R.
Acrylic resins for dentures. J Am Dent Assoc, v. 29, n. 1, p. 7-33, Jan. 1942.

26. TAKAMATA, T.; SETCOS, J. C. Resin denture bases: review of accuracy and methods of polymerization. Int $\mathbf{J}$ Prosthod, v. 2, n. 6, p. 555-562, Dec. 1989.

27. TAKAMATA, T.; SETCOS, J. C.; PHILlIPS, R. W. et al. Adaptation of acrylic resin dentures as influenced by the activation mode of polymerization. J Am Dent Assoc, v. 119, n. 2, p. 271-276, Aug. 1989.

28. WINKLER, S.; ORTMAN, H. R.; MORRIS, H. F. et al. Processing changes in complete dentures constructed from pour resins. J Am Dent Assoc, v. 82, n. 2, p. 349-353, Feb. 1971

29. WOELFEL, J. B.; PAFFENBARGER, G. C.; SWEENEY, W. T. Clinical evaluation of complete dentures mode of 11 different types of denture base materials. J Am Dent Assoc, v. 70, n. 5, p. 1170-1188, May 1965.

30. WOLFAARDT, J.; CLEATON-JONES, P.; FATTI, P. The influence of processing variables on dimensional changes of heat-cured polymethylmethacrylate. J Prosthet Dent, v. 55, n. 4, p. 518-525, Apr. 1986.

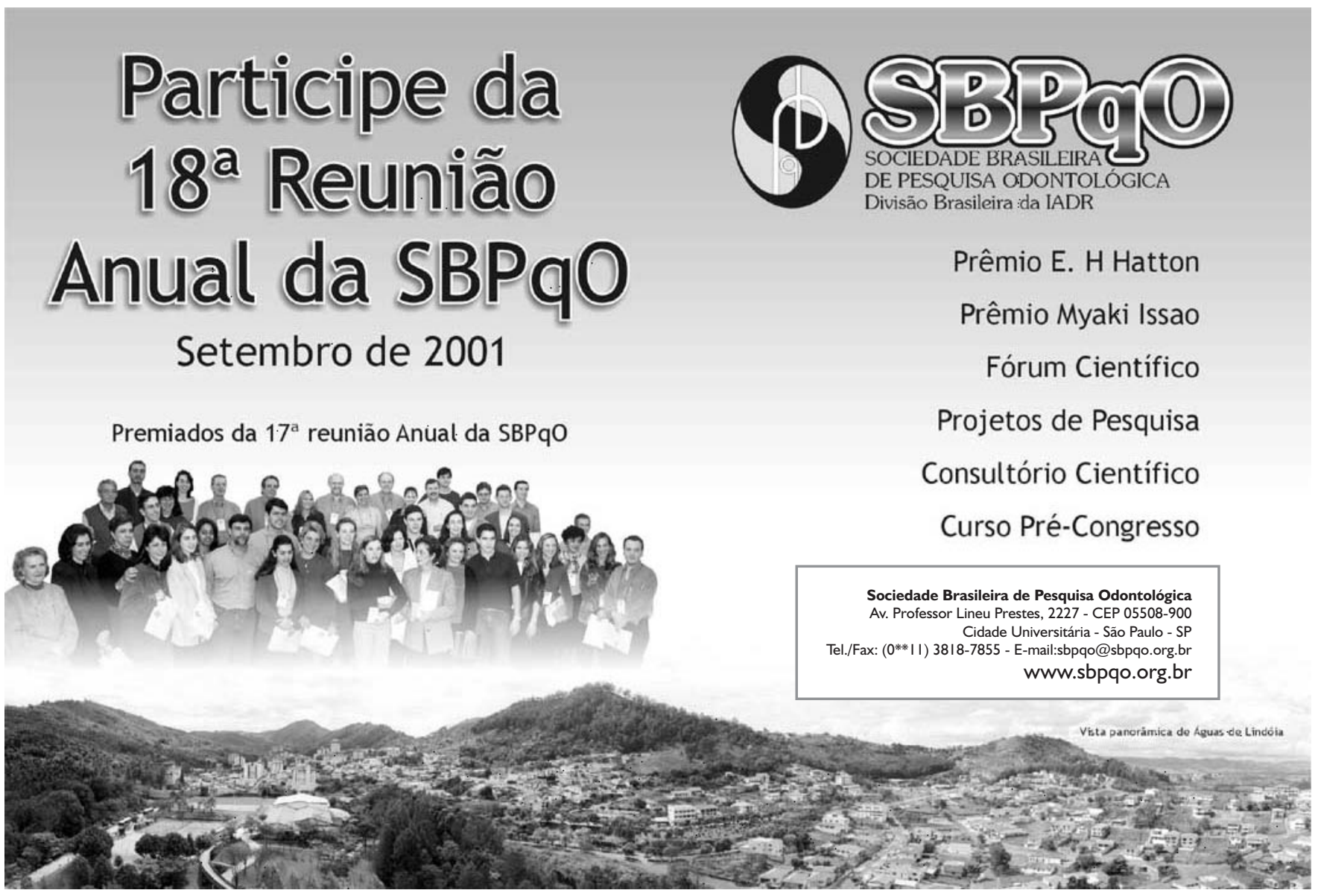

